\title{
Sato-Tate, cyclicity, and divisibility statistics on average for elliptic curves of small height
}

\author{
WILLIAM D. BANKS \\ Department of Mathematics, University of Missouri \\ Columbia, MO 65211 USA \\ bbanks@math.missouri.edu \\ IGOR E. SHPARLINSKI \\ Department of Computing, Macquarie University \\ Sydney, NSW 2109, Australia \\ igor@ics.mq.edu.au
}

March 6, 2018

\begin{abstract}
We obtain asymptotic formulae for the number of primes $p \leqslant x$ for which the reduction modulo $p$ of the elliptic curve

$$
\mathbf{E}_{a, b}: Y^{2}=X^{3}+a X+b
$$

satisfies certain "natural" properties, on average over integers $a$ and $b$ such that $|a| \leqslant A$ and $|b| \leqslant B$, where $A$ and $B$ are small relative to $x$. More precisely, we investigate behavior with respect to the Sato-Tate conjecture, cyclicity, and divisibility of the number of points by a fixed integer $m$.
\end{abstract}




\section{Introduction}

\subsection{Motivation}

For integers $a$ and $b$ such that $4 a^{3}+27 b^{2} \neq 0$, we denote by $\mathbf{E}_{a, b}$ the elliptic curve defined by the affine Weierstraß equation:

$$
\mathbf{E}_{a, b}: Y^{2}=X^{3}+a X+b .
$$

For a basic background on elliptic curves, we refer the reader to the book 44 by Silverman.

For any prime $p>3$, we denote by $\mathbb{F}_{p}$ the finite field with $p$ elements, which we identify with the set of integers $\{0, \pm 1, \ldots, \pm(p-1) / 2\}$.

When $p \nmid 4 a^{3}+27 b^{2}$, the set $\mathbf{E}_{a, b}\left(\mathbb{F}_{p}\right)$ consisting of the $\mathbb{F}_{p}$-rational points of $\mathbf{E}_{a, b}$ together with a point at infinity forms an abelian group under an appropriate composition rule called addition, and the number of elements in the group $\mathbf{E}_{a, b}\left(\mathbb{F}_{p}\right)$ satisfies the Hasse bound:

$$
\left|\# \mathbf{E}_{a, b}\left(\mathbb{F}_{p}\right)-p-1\right| \leqslant 2 \sqrt{p}
$$

(see, for example, [44, Chapter V, Theorem 1.1]).

A well known conjecture in the theory of elliptic curves, known as the Lang-Trotter conjecture (see [35]), asserts that for any elliptic curve $\mathbf{E}_{a, b}$ and any fixed integer $t$, the number $\pi_{a, b}(t ; x)$ of primes $p \leqslant x$ (with $\left.p \nmid 4 a^{3}+27 b^{2}\right)$ such that

$$
\# \mathbf{E}_{a, b}\left(\mathbb{F}_{p}\right)=p+1-t
$$

satisfies the asymptotic formula

$$
\pi_{a, b}(t ; x) \sim c_{a, b, t} \cdot \frac{\sqrt{x}}{\log x} \quad(x \rightarrow \infty)
$$

with some constant $c_{a, b, t}$ that depends only on $a, b$, and $t$, provided that $\mathbf{E}_{a, b}$ does not have complex multiplication (see [44, Section III.4]) or $t$ is nonzero. The Lang-Trotter conjecture remains open, although some progress has been made (see the survey [40]).

Fouvry and Murty [23] have studied the problem of estimating $\pi_{a, b}(0 ; x)$ on average over integers $a, b$ with $|a| \leqslant A$ and $|b| \leqslant B$ and have shown (see [23, Theorem 6]) that the asymptotic formula

$$
\frac{1}{4 A B} \sum_{|a| \leqslant A} \sum_{|b| \leqslant B} \pi_{a, b}(0 ; x) \sim \frac{\pi}{3} \cdot \frac{\sqrt{x}}{\log x} \quad(x \rightarrow \infty)
$$


holds uniformly in the range

$$
A B \geqslant x^{3 / 2+\varepsilon} \quad \text { and } \quad \min \{A, B\} \geqslant x^{1 / 2+\varepsilon},
$$

where $\varepsilon>0$ is fixed. For the case $t \neq 0$, David and Pappalardi [19] have established the following asymptotic formula in a shorter range of $A$ and $B$ :

$$
\frac{1}{4 A B} \sum_{|a| \leqslant A} \sum_{|b| \leqslant B} \pi_{a, b}(t ; x) \sim C_{t} \cdot \frac{\sqrt{x}}{\log x} \quad(x \rightarrow \infty),
$$

where

$$
C_{t}=\frac{2}{\pi} \prod_{p \mid t}\left(1-\frac{1}{p^{2}}\right)^{-1} \prod_{p \nmid t} \frac{p\left(p^{2}-p-1\right)}{(p-1)\left(p^{2}-1\right)} .
$$

This work has been extended by Baier [3] to the full range (11). Other results along these lines have been obtained in [1, 4, 9, 20, 26, 32, 33].

We remark that another type of problem "on average" over a similar family of curves has been considered in [21, 27].

Here, we investigate the average behavior of the family of curves $\mathbf{E}_{a, b}$ with $|a| \leqslant A$ and $|b| \leqslant B$ with respect to some natural statistical properties of their reductions modulo $p$. Although these properties are expected to hold for individual curves, such results remain inaccessible.

\subsection{Our Results}

In the present paper, we study how the family of curves $\mathbf{E}_{a, b}$ with $|a| \leqslant A$ and $|b| \leqslant B$ behaves with respect to:

- the Sato-Tate conjecture about the distribution of the cardinalities $\# \mathbf{E}_{a, b}\left(\mathbb{F}_{p}\right)($ see $[34]) ;$

- cyclicity of the group $\mathbf{E}_{a, b}\left(\mathbb{F}_{p}\right)$, a notion which essentially dates back to the work of Borosh, Moreno and Porta [11] and of Serre [43];

- divisibility of $\# \mathbf{E}_{a, b}\left(\mathbb{F}_{p}\right)$ by a given integer $m$.

Accordingly, for real $0 \leqslant \alpha<\beta \leqslant \pi$, we define the Sato-Tate density

$$
\mu_{\mathrm{ST}}(\alpha, \beta)=\frac{2}{\pi} \int_{\alpha}^{\beta} \sin ^{2} \theta d \theta,
$$


and we define the angle $\psi_{a, b}(p) \in[0, \pi]$ via the identity

$$
p+1-\# \mathbf{E}_{a, b}\left(\mathbb{F}_{p}\right)=2 \sqrt{p} \cos \psi_{a, b}(p) .
$$

We denote by $\Pi_{a, b}^{\mathrm{ST}}(\alpha, \beta ; x)$ the number of primes $p \leqslant x$ (with $\left.p \nmid 4 a^{3}+27 b^{2}\right)$ for which $\alpha \leqslant \psi_{a, b}(p) \leqslant \beta$. The Sato-Tate conjecture asserts that if $\mathbf{E}_{a, b}$ does not have complex multiplication, then the asymptotic formula

$$
\Pi_{a, b}^{\mathrm{ST}}(\alpha, \beta ; x) \sim \mu_{\mathrm{ST}}(\alpha, \beta) \cdot \frac{x}{\log x} \quad(x \rightarrow \infty)
$$

holds (see [10, 34, 41]).

It is well known that $\mathbf{E}_{a, b}\left(\mathbb{F}_{p}\right)$ is an abelian group of rank at most two. We denote by $\Pi_{a, b}^{\mathrm{C}}(x)$ the number of primes $p \leqslant x$ (with $p \nmid 4 a^{3}+27 b^{2}$ ) for which $\mathbf{E}_{a, b}\left(\mathbb{F}_{p}\right)$ is cyclic. The conjectured asymptotic formula

$$
\Pi_{a, b}^{\mathrm{C}}(x) \sim C_{a, b} \cdot \frac{x}{\log x} \quad(x \rightarrow \infty),
$$

where $C_{a, b}$ is a constant that depends only on $a$ and $b$, has been established conditionally (under the Extended Riemann Hypothesis) in some cases, and there are several unconditional lower bounds on $\Pi_{a, b}^{\mathrm{C}}(x)$; see the original papers [13, 14, 17, 18, 28, 39, 43] as well as the recent surveys [15, 40].

Finally, for a fixed integer $m \geqslant 1$, we denote by $\Pi_{a, b}^{\mathrm{D}}(m ; x)$ the number of primes $p \leqslant x$ (with $p \nmid 4 a^{3}+27 b^{2}$ ) for which $m \mid \# \mathbf{E}_{a, b}\left(\mathbb{F}_{p}\right)$.

It worth mentioning that the Chebotarev density theorem can be used to study $\Pi_{a, b}^{\mathrm{D}}(m ; x)$ for individual curves (see [15]). By averaging over $a$ and $b$, however, we obtain sharper results which are also uniform in $m$ up to any fixed power of $\log x$.

Taylor [45] has recently announced a complete proof of the Sato-Tate conjecture, which implies (2) in particular, but his work on individual curves does not imply any results on average due to the lack of uniformity with respect to the coefficients $a$ and $b$ in the Weierstraß equation.

Here, we obtain an asymptotic formula for the number of pairs $(a, b)$ with $|a| \leqslant A,|b| \leqslant B$ and $p \nmid 4 a^{3}+27 b^{2}$ such that $\mathbf{E}_{a, b}\left(\mathbb{F}_{p}\right)$ belongs to a certain sufficiently "massive" collection of isomorphism classes of elliptic 
curves. Using this result, we derive asymptotic formulae for the sums

$$
\begin{aligned}
& N_{\alpha, \beta}^{\mathrm{ST}}(A, B ; x)=\sum_{|a| \leqslant A} \sum_{|b| \leqslant B} \Pi_{a, b}^{\mathrm{ST}}(\alpha, \beta ; x), \\
& N^{\mathrm{C}}(A, B ; x)=\sum_{|a| \leqslant A} \sum_{|b| \leqslant B} \Pi_{a, b}^{\mathrm{C}}(x), \\
& N_{m}^{\mathrm{D}}(A, B ; x)=\sum_{|a| \leqslant A} \sum_{|b| \leqslant B} \Pi_{a, b}^{\mathrm{D}}(m ; x) .
\end{aligned}
$$

The main terms in our expansions of $N_{\alpha, \beta}^{\mathrm{ST}}(A, B ; x), N^{\mathrm{C}}(A, B ; x)$ and $N_{m}^{\text {D }}(A, B ; x)$ are derived from asymptotic formulae of Birch [10], Vlădut [46] and Howe [29], respectively. The asymptotic formula of Birch [10] is not quite sufficient for our purposes, however, so we give an explicit bound for the error term which is obtained using the method of Niederreiter [42].

In the case of the Sato-Tate distribution, the computation of the error term is almost trivial. The other cases require a more specialized treatment, and these are done using now standard techniques; for example, we apply a result of Indlekofer, Wehmeier and Lucht [30].

In the last section, we give an outline of several other questions concerning reductions of elliptic curves that can be approached with our method.

\subsection{Our Method}

The functions $N_{\alpha, \beta}^{\mathrm{ST}}(A, B ; x), N^{\mathrm{C}}(A, B ; x)$ and $N_{m}^{\mathrm{D}}(A, B ; x)$ can be studied via the method of Fouvry and Murty [23], which makes essential use of the Weil bound; see [37, Chapter 5]. Here, however, we obtain sharper results by applying bounds on multiplicative character sums rather than estimating exponential sums as in 23]. Using the Polya-Vinogradov and Burgess bounds (see [31, Theorems 12.5 and 12.6]) one already obtains stronger results for individual primes than with exponential sums. Moreover, the use of multiplicative character sums allows for additional savings on average over primes $p \leqslant x$. In the present paper, we use a result of Garaev [25] on multiplicative character sums, which is derived from a variant of the large sieve inequality (see [31, Section 7.4]), and we obtain nontrivial bounds in a region that is significantly wider than (1). Our method leads to bounds which are nontrivial whenever $A$ and $B$ satisfy the inequalities

$$
A, B \geqslant x^{\varepsilon} \quad \text { and } \quad A B \geqslant x^{1+\varepsilon} .
$$


However, to avoid some tedious technical complications and more cluttered expressions for the error term, we further assume that

$$
A, B \leqslant x^{1-\varepsilon}
$$

(which of course makes the first inequality in (3) redundant). Certainly, the case in which $A$ and $B$ are both small is of primary interest, so the restriction (4) is rather mild.

One of the main ingredients of the method of Fouvry and Murty is the use of the Weil bound to prove the asymptotic formula $2 A B / p+O\left(p^{1 / 2+o(1)}\right)$ for the number of curves $\mathbf{E}_{a, b}$ with $|a| \leqslant A \leqslant(p-1) / 2$ and $|b| \leqslant B \leqslant(p-1) / 2$ that are isomorphic to a given curve $\mathbf{E}_{r, s}$; see [23, Section 7]. Here, we show that, on average over $r$ and $s$, the error term can be improved substantially, and this suffices for the problems that we consider. On the other hand, our method does not directly apply to the question considered in [23] since a set of elliptic curves over $\mathbb{F}_{p}$ with a prescribed number of $\mathbb{F}_{p}$-rational points (that is, a set of isogenous curves) is much "thinner" than the sets of curves with which we work. Of course, there is some possibility that both approaches might be combined to improve the threshold (11) for the original problem.

Baier and Zhao [5] have also studied the distribution of $N_{\alpha, \beta}^{\mathrm{ST}}(A, B ; x)$ using a very different method from ours; their results are also different (but there is partial overlap) and in many cases are weaker with respect to the range of $A$ and $B$ as well as the uniformity in $\alpha$ and $\beta$. In particular, among other restrictions, the inequalities

$$
A, B \geqslant x^{1 / 2+\varepsilon}
$$

are required for [5, Theorem 1]. In some cases, however, the results of [5] are stronger than ours. It is worth mentioning that Baier and Zhao [5] have estimated the average deviation of $\Pi_{a, b}^{\mathrm{ST}}(\alpha, \beta ; x)$ from the value predicted by the Sato-Tate conjecture.

Finally, several more results in these directions have recently been obtained in [4] that also appear to be weaker than our results.

\subsection{Notation}

Throughout the paper, any implied constants in the symbols $O$ and $\ll$ may occasionally depend, where obvious, on the parameters $\varepsilon$ and $K$ but are absolute otherwise. We recall that the notations $U \ll V$ and $U=O(V)$ are 
both equivalent to the statement that the inequality $|U| \leqslant c V$ holds with some constant $c>0$.

The letters $p$ and $q$ always denote prime numbers, while $m$ and $n$ always denote integers. As usual, we use $\pi(x)$ to denote the number of primes $p \leqslant x$.

\subsection{Acknowledgements}

The authors are grateful to Antal Balog for fruitful discussions which have led to an improvement of our original results. The authors would also like to thank Nick Katz for several comments concerning the Sato-Tate conjecture and in particular for his suggestion of Lemma 9. This work began during a pleasant visit by W. B. to Macquarie University; the support and hospitality of this institution are gratefully acknowledged. During the preparation of this paper, I. S. was supported in part by ARC grant DP0556431.

\section{Preliminaries}

\subsection{Character sums}

For a prime $p$, we denote by $\mathcal{X}_{p}$ the set of multiplicative characters of $\mathbb{F}_{p}$, $\chi_{0}$ the principal character of $\mathbb{F}_{p}$, and $\mathcal{X}_{p}^{*}=\mathcal{X}_{p} \backslash\left\{\chi_{0}\right\}$ the set of nonprincipal characters; we refer the reader to [31, Chapter 3] for the necessary background on multiplicative characters. We recall the following orthogonality relations:

$$
\frac{1}{p-1} \sum_{\chi \in \mathcal{X}_{p}} \chi(v)= \begin{cases}1 & \text { if } v=1 \\ 0 & \text { otherwise }\end{cases}
$$

and

$$
\frac{1}{p-1} \sum_{u \in \mathbb{F}_{p}^{*}} \chi_{1}(u) \bar{\chi}_{2}(u)= \begin{cases}1 & \text { if } \chi_{1}=\chi_{2} \\ 0 & \text { otherwise }\end{cases}
$$

for all $v \in \mathbb{F}_{p}$ and $\chi_{1}, \chi_{2} \in \mathcal{X}_{p}$ (here, $\bar{\chi}_{2}$ is the character obtained from $\chi_{2}$ by complex conjugation).

The following result combines the Polya-Vinogradov bound (for $\nu=1$ ) with the Burgess bounds (for $\nu \geqslant 2$ ); see [31, Bound (12.58)] and also [31, Theorems 12.5 and 12.6]: 
Lemma 1. Uniformly for all primes $p$, all positive integers $L, M, \nu$, and all characters $\chi \in \mathcal{X}_{p}^{*}$, we have

$$
\sum_{n=L+1}^{L+M} \chi(n) \ll M^{1-1 / \nu} p^{(\nu+1) /\left(4 \nu^{2}\right)}(\log p)^{1 / \nu} .
$$

The next bound is due to Ayyad, Cochrane and Zheng [2, Theorem 2]; see also the result of Friedlander and Iwaniec [24]:

Lemma 2. Uniformly for all positive integers $L, M$, we have

$$
\sum_{\chi \in \mathcal{X}_{p}^{*}}\left|\sum_{n=L+1}^{L+M} \chi(n)\right|^{4} \ll p M^{2+o(1)} \quad(p \rightarrow \infty) .
$$

We also need the following statement, which is contained in the more general result [25, Theorem 10] of Garaev (which also applies to character sums with composite moduli and allows significantly more flexibility in the choice of $M)$ :

Lemma 3. Fix $\varepsilon>0$ and $\eta>0$. If $x$ is sufficiently large, then for all $M \geqslant x^{\varepsilon}$, all primes $p \leqslant x$ with at most $x^{3 / 4+4 \eta+o(1)}$ exceptions as $x \rightarrow \infty$, and all characters $\chi \in \mathcal{X}_{p}^{*}$, we have

$$
\left|\sum_{n=1}^{M} \chi(n)\right| \leqslant M^{1-\eta}
$$

where the function implied by o(1) depends only on $\varepsilon$ and $\eta$.

Proof. We can assume that $\eta<1 / 16$ for otherwise there is nothing to prove.

If $M \geqslant x^{3 / 4}$, then the result follows from Lemma 1 with $\nu=1$. Indeed, for a prime $p \leqslant x$ we have

$$
\left|\sum_{n=1}^{M} \chi(n)\right| \leqslant p^{1 / 2+o(1)} \leqslant x^{1 / 2+o(1)} \leqslant M^{2 / 3+o(1)} \leqslant M^{1-\eta}
$$

for any fixed $\eta \in(0,1 / 16)$ if $x$ is large enough.

For $M \leqslant x^{3 / 4}$ the result is a direct consequence of [25, Theorem 10]. 


\subsection{Distribution of powers}

Let $d_{p}=\operatorname{gcd}(p-1,6)$ and put

$$
\sigma_{p}(M)=\max _{\substack{\chi \in \mathcal{X}_{p}^{*} \\ \chi^{d_{p}=\chi_{0}}}}\left\{1,\left|\sum_{n=1}^{M} \chi(n)\right|\right\}
$$

For any integers $B, s$ we define

$$
\mathcal{Z}_{s}(B ; p)=\left\{u \in \mathbb{F}_{p}^{*}: s u^{6} \equiv b \quad(\bmod p) \text { where }|b| \leqslant B\right\} .
$$

We have the following bound on the cardinality of $\mathcal{Z}_{s}(B ; p)$ :

Lemma 4. For all primes $p$ and all positive integers $B, s<p$, we have

$$
\left|\# \mathcal{Z}_{s}(B ; p)-2 B\right| \leqslant 11 \sigma_{p}(B)
$$

Proof. For all $n \in \mathbb{Z}$ we have

$$
\#\left\{u \in \mathbb{F}_{p}^{*}: u^{6} \equiv n \quad(\bmod p)\right\}=\sum_{\substack{\chi \in \mathcal{X}_{p} \\ \chi^{d_{p}}=\chi_{0}}} \chi(n) .
$$

If $\bar{s}$ is an integer such that $s \bar{s} \equiv 1(\bmod p)$, it follows that

$$
\# \mathcal{Z}_{s}(B ; p)=\sum_{|b| \leqslant B} \sum_{\substack{\chi \in \mathcal{X}_{p} \\ \chi^{d_{p}}=\chi_{0}}} \chi(\bar{s} b)=(2 B+1)+\sum_{\substack{\chi \in \mathcal{X}_{p}^{*} \\ \chi^{d_{p}}=\chi_{0}}} \bar{\chi}(s) \sum_{|b| \leqslant B} \chi(b) .
$$

Since the inner sum is bounded by

$$
\left|\sum_{|b| \leqslant B} \chi(b)\right| \leqslant 2 \sigma_{p}(B)
$$

and

$$
\#\left\{\chi \in \mathcal{X}_{p}^{*}: \chi^{d_{p}}=\chi_{0}\right\}=d_{p}-1 \leqslant 5,
$$

the result follows.

For any integers $A, B, r, s$ we define

$$
\mathcal{Z}_{r, s}(A, B ; p)=\left\{u \in \mathcal{Z}_{s}(B ; p): r u^{4} \equiv a \quad(\bmod p) \text { where }|a| \leqslant A\right\} .
$$


Lemma 5. For all primes $p$ and all positive integers $A, B, s<p$, we have

$\sum_{r \in \mathbb{F}_{p}}\left|\# \mathcal{Z}_{r, s}(A, B ; p)-\frac{2 A \cdot \# \mathcal{Z}_{s}(B ; p)}{p}\right| \leqslant A^{1 / 2} B p^{1 / 4+o(1)}+A^{1 / 2} B^{1 / 2} p^{1 / 2+o(1)}$ as $p \rightarrow \infty$.

Proof. We can assume that $A B>p$ since the result is trivial otherwise. Indeed, if $A B \leqslant p$ then $A^{1 / 2} B^{1 / 2} p^{1 / 2} \geqslant A B$, while

$$
\begin{aligned}
\sum_{r \in \mathbb{F}_{p}}\left|\# \mathcal{Z}_{r, s}(A, B ; p)-\frac{2 A \cdot \# \mathcal{Z}_{s}(B ; p)}{p}\right| \\
\quad \leqslant \sum_{r \in \mathbb{F}_{p}} \# \mathcal{Z}_{r, s}(A, B ; p)+2 A \cdot \# \mathcal{Z}_{s}(B ; p) \ll A B .
\end{aligned}
$$

For every $a \in \mathbb{F}_{p}^{*}$ let $\bar{a}$ be an integer such that $a \bar{a} \equiv 1(\bmod p)$. Using (5) it follows that

$$
\begin{aligned}
\# \mathcal{Z}_{r, s}(A, B ; p) & =\sum_{u \in \mathcal{Z}_{s}(B ; p)} \sum_{0<|a| \leqslant A} \frac{1}{p-1} \sum_{\chi \in \mathcal{X}_{p}} \chi\left(r u^{4} \bar{a}\right) \\
& =\frac{2 A \cdot \# \mathcal{Z}_{s}(B ; p)}{p-1}+O(1)+\frac{1}{p-1} \sum_{\chi \in \mathcal{X}_{p}^{*}} \chi(r) \sum_{u \in \mathcal{Z}_{s}(B ; p)} \chi\left(u^{4}\right) \sum_{|a| \leqslant A} \bar{\chi}(a) .
\end{aligned}
$$

Since

$$
\frac{2 A \cdot \# \mathcal{Z}_{s}(B ; p)}{p}-\frac{2 A \cdot \# \mathcal{Z}_{s}(B ; p)}{p-1} \ll \frac{A \cdot \# \mathcal{Z}_{s}(B ; p)}{p^{2}} \ll \frac{A B}{p^{2}} \ll 1,
$$

we have

$$
\sum_{r \in \mathbb{F}_{p}}\left|\# \mathcal{Z}_{r, s}(A, B ; p)-\frac{2 A \cdot \# \mathcal{Z}_{s}(B ; p)}{p}\right| \ll p+W
$$

where

$$
W=\frac{1}{p} \sum_{r \in \mathbb{F}_{p}}\left|\sum_{\chi \in \mathcal{X}_{p}^{*}} \chi(r) \sum_{u \in \mathcal{Z}_{s}(B ; p)} \chi\left(u^{4}\right) \sum_{|a| \leqslant A} \bar{\chi}(a)\right| .
$$

By the Cauchy inequality, $W^{2}$ does not exceed

$$
\begin{aligned}
& \frac{1}{p} \sum_{r \in \mathbb{F}_{p}}\left|\sum_{\chi \in \mathcal{X}_{p}^{*}} \chi(r) \sum_{u \in \mathcal{Z}_{s}(B ; p)} \chi\left(u^{4}\right) \sum_{|a| \leqslant A} \bar{\chi}(a)\right|^{2} \\
& \quad=\frac{1}{p} \sum_{\chi_{1}, \chi_{2} \in \mathcal{X}_{p}^{*}} \sum_{u_{1}, u_{2} \in \mathcal{Z}_{s}(B ; p)} \chi_{1}\left(u_{1}^{4}\right) \bar{\chi}_{2}\left(u_{2}^{4}\right) \sum_{\left|a_{1}\right|,\left|a_{2}\right| \leqslant A} \bar{\chi}_{1}\left(a_{1}\right) \chi_{2}\left(a_{2}\right) \sum_{r \in \mathbb{F}_{p}} \chi_{1}(r) \bar{\chi}_{2}(r) .
\end{aligned}
$$


Using the orthogonality relation (6) we deduce that

$$
W^{2} \leqslant\left.\sum_{\chi \in \mathcal{X}_{p}^{*}}\left|\sum_{u \in \mathcal{Z}_{s}(B ; p)} \chi\left(u^{4}\right)\right|^{2}|| \sum_{|a| \leqslant A} \chi(a)\right|^{2} .
$$

Applying the Cauchy inequality again, it follows that

$$
W^{4} \leqslant \sum_{\chi \in \mathcal{X}_{p}^{*}}\left|\sum_{u \in \mathcal{Z}_{s}(B ; p)} \chi\left(u^{4}\right)\right|^{4} \cdot \sum_{\chi \in \mathcal{X}_{p}^{*}}\left|\sum_{|a| \leqslant A} \chi(a)\right|^{4} .
$$

The second sum is of size $O\left(p^{1+o(1)} A^{2}\right)$ by Lemma 2, For the first sum, we extend the summation to include the trivial character $\chi=\chi_{0}$, obtaining

$$
\sum_{\chi \in \mathcal{X}_{p}^{*}}\left|\sum_{u \in \mathcal{Z}_{s}(B ; p)} \chi\left(u^{4}\right)\right|^{4} \leqslant \sum_{\chi \in \mathcal{X}_{p}}\left|\sum_{u \in \mathcal{Z}_{s}(B ; p)} \chi\left(u^{4}\right)\right|^{4}=p T,
$$

where $T$ is the number of solutions to the congruence

$$
u_{1}^{4} u_{2}^{4} \equiv u_{3}^{4} u_{4}^{4} \quad(\bmod p), \quad u_{1}, u_{2}, u_{3}, u_{4} \in \mathcal{Z}_{s}(B ; p) .
$$

Note that $T$ does not exceed the number of quadruples $\left(u_{1}, u_{2}, u_{3}, u_{4}\right)$ in $\mathcal{Z}_{s}(B ; p)^{4}$ for which

$$
u_{1}^{12} u_{2}^{12} \equiv u_{3}^{12} u_{4}^{12} \quad(\bmod p) .
$$

Since $s u_{j}^{6} \equiv b_{j}(\bmod p)$ for some $b_{j}$ with $\left|b_{j}\right| \leqslant B$, and each $b_{j}$ corresponds to at most six values of $u_{j}$, it follows that $T \leqslant 6^{4} R$, where $R$ is the number of solutions to the congruence

$$
b_{1}^{2} b_{2}^{2} \equiv b_{3}^{2} b_{4}^{2} \quad(\bmod p), \quad\left|b_{1}\right|,\left|b_{2}\right|,\left|b_{3}\right|,\left|b_{4}\right| \leqslant B
$$

Clearly, $R=2 Q$, where $Q$ is the number of solutions to the congruence

$$
b_{1} b_{2} \equiv b_{3} b_{4} \quad(\bmod p), \quad\left|b_{1}\right|,\left|b_{2}\right|,\left|b_{3}\right|,\left|b_{4}\right| \leqslant B .
$$

Writing

$$
Q=\frac{1}{p-1} \sum_{\chi \in \mathcal{X}_{p}}\left|\sum_{|b| \leqslant B} \chi(b)\right|^{4}=\frac{(2 B+1)^{4}}{p-1}+\frac{1}{p-1} \sum_{\chi \in \mathcal{X}_{p}^{*}}\left|\sum_{|b| \leqslant B} \chi(b)\right|^{4}
$$


and using Lemma 2 again, we see that

$$
T \ll R \ll Q \ll B^{4} p^{-1}+B^{2} p^{o(1)} .
$$

Collecting the above estimates and substituting them into (10) we deduce that

$$
W^{4} \ll p^{2+o(1)} A^{2}\left(B^{4} p^{-1}+B^{2}\right)
$$

which together with (8) implies that

$$
\begin{aligned}
& \sum_{r \in \mathbb{F}_{p}}\left|\# \mathcal{Z}_{r, s}(A, B ; p)-\frac{2 A \cdot \# \mathcal{Z}_{s}(B ; p)}{p}\right| \\
& \ll p+A^{1 / 2} B p^{1 / 4+o(1)}+A^{1 / 2} B^{1 / 2} p^{1 / 2+o(1)} .
\end{aligned}
$$

Finally, for $A B>p$ we have $p<A^{1 / 2} B^{1 / 2} p^{1 / 2}$, and the result follows.

Combining Lemmas 4 and 5 we immediately obtain:

Corollary 6. For all primes $p$ and all positive integers $A, B, s<p$, we have

$$
\begin{aligned}
& \sum_{r \in \mathbb{F}_{p}}\left|\# \mathcal{Z}_{r, s}(A, B ; p)-\frac{4 A B}{p}\right| \\
& \ll A \sigma_{p}(B)+A^{1 / 2} B p^{1 / 4+o(1)}+A^{1 / 2} B^{1 / 2} p^{1 / 2+o(1)} .
\end{aligned}
$$

For large values of $A$, the following lemma provides a stronger bound for the sum considered in Lemma 5 ,

Lemma 7. For all primes $p$ and all positive integers $A, B, s<p$, we have

$$
\sum_{r \in \mathbb{F}_{p}}\left|\# \mathcal{Z}_{r, s}(A, B ; p)-\frac{2 A \cdot \# \mathcal{Z}_{s}(B ; p)}{p}\right| \ll B^{1 / 2} p \log p .
$$

Proof. We argue as in the proof of Lemma 5 arriving at (91). Next, we apply Lemma 1 with $\nu=1$ (that is, the Polya-Vinogradov bound) followed by the Cauchy inequality, deriving the bound

$$
\begin{aligned}
W^{2} & \ll p(\log p)^{2} \sum_{\chi \in \mathcal{X}_{p}^{*}}\left|\sum_{u \in \mathcal{Z}_{s}(B ; p)} \chi\left(u^{4}\right)\right|^{2} \\
& \leqslant p(\log p)^{2} \sum_{\chi \in \mathcal{X}_{p}}\left|\sum_{u \in \mathcal{Z}_{s}(B ; p)} \chi\left(u^{4}\right)\right|^{2}=p(p-1)(\log p)^{2} T
\end{aligned}
$$


where $T$ is the number of solutions to the congruence

$$
u_{1}^{4} \equiv u_{2}^{4} \quad(\bmod p), \quad u_{1}, u_{2} \in \mathcal{Z}_{s}(B ; p) .
$$

Since $T \leqslant 4 \# \mathcal{Z}_{s}(B ; p)=O(B)$, the result follows.

We remark that for in the proof of Lemma 7 one can use Lemma 1 with values of $\nu$ other than one, but doing so does not lead to any improvement over the bound of Lemma 5.

Combining Lemmas 4 and 7 we obtain:

Corollary 8. For all primes $p$ and all positive integers $A, B, s<p$, we have

$$
\sum_{r \in \mathbb{F}_{p}}\left|\# \mathcal{Z}_{r, s}(A, B ; p)-\frac{4 A B}{p}\right| \ll A \sigma_{p}(B)+B^{1 / 2} p \log p .
$$

\subsection{Statistics of elliptic curves}

It is well known that if $a, b, r, s \in \mathbb{F}_{p}$, then the two curves $\mathbf{E}_{a, b}$ and $\mathbf{E}_{r, s}$ are isomorphic over $\mathbb{F}_{p}$ if and only if $a=r u^{4}$ and $b=s u^{6}$ for some $u \in \mathbb{F}_{p}^{*}$. In particular, each curve $\mathbf{E}_{a, b}$ with $a, b \in \mathbb{F}_{p}^{*}$ is isomorphic to $(p-1) / 2$ elliptic curves $\mathbf{E}_{r, s}$, and there are $2 p+O(1)$ distinct isomorphism classes of elliptic curves over $\mathbb{F}_{p}$; see [36]. Thus, our results can be conveniently formulated in terms of counting functions for individual curves $\mathbf{E}_{a, b}$ rather than in terms of isomorphism classes of curves, as in the papers [10, 29, 46].

Let $\mathcal{T}_{p}(\alpha, \beta)$ be the set of set of pairs $(a, b) \in \mathbb{F}_{p}^{*} \times \mathbb{F}_{p}^{*}$ such that the inequalities $\alpha \leqslant \psi_{a, b}(p) \leqslant \beta$ hold. Thanks to Birch [10], one knows that

$$
\# \mathcal{T}_{p}(\alpha, \beta) \sim \mu_{\mathrm{ST}}(\alpha, \beta) p^{2} \quad(p \rightarrow \infty),
$$

however we require a stronger result. What is needed is a full analogue for the Sato-Tate density of the bound of Niederreiter [42] on the discrepancy in the distribution of values of (normalized) Kloosterman sums. Fortunately, such a result can be obtained using the same methods since all of the underlying tools, namely [42, Lemma 3] and [34, Theorem 13.5.3], apply to $\psi_{a, b}(p)$ as well as to values of Kloosterman sums. In particular, from [34, Theorem 13.5.3] it follows that

$$
\frac{1}{(q-1)^{2}} \sum_{\substack{a, b \in \mathbb{F}_{p}^{*} \\ 4 a^{3}+27 b^{2} \neq 0}} \frac{\sin \left((n+1) \psi_{a, b}(p)\right)}{\sin \left(\psi_{a, b}(p)\right)} \ll n q^{-1 / 2} \quad(n=1,2, \ldots)
$$

(see also the work of Fisher [22, Section 5]). Thus, as in [42], we have: 
Lemma 9. Uniformly for all primes $p$, we have

$$
\max _{0 \leqslant \alpha<\beta \leqslant \pi}\left|\# \mathcal{T}_{p}(\alpha, \beta)-\mu_{\mathrm{ST}}(\alpha, \beta) p^{2}\right| \ll p^{7 / 4} .
$$

Next, for any prime $p$ we denote

$$
\vartheta_{p}=\prod_{q \mid p-1}\left(1-\frac{1}{q\left(q^{2}-1\right)}\right)
$$

where the product is taken over all prime divisors $q$ of $p-1$.

Let $\mathcal{C}_{p}$ be the set of pairs $(a, b) \in \mathbb{F}_{p}^{*} \times \mathbb{F}_{p}^{*}$ such that $\mathbf{E}_{a, b}\left(\mathbb{F}_{p}\right)$ is cyclic. The cardinality of $\mathcal{C}_{p}$ has been estimated by Vlăduţ [46] as follows:

Lemma 10. For all primes $p$, we have

$$
\left|\# \mathcal{C}_{p}-\vartheta_{p} p^{2}\right| \leqslant p^{3 / 2+o(1)} \quad(p \rightarrow \infty) .
$$

Finally, for any integer $k$, let $\omega_{k}(m)$ denote the completely multiplicative function which is defined on prime powers $q^{j}$ as follows:

$$
\omega_{k}\left(q^{j}\right)= \begin{cases}\frac{1}{q^{j-1}(q-1)} & \text { if } k \neq 1 \quad\left(\bmod q^{\lceil j / 2\rceil}\right) ; \\ \frac{q^{\lfloor j / 2\rfloor+1}+q^{\lfloor j / 2\rfloor}-1}{q^{j+\lfloor j / 2\rfloor-1}\left(q^{2}-1\right)} & \text { if } k \equiv 1 \quad\left(\bmod q^{\lceil j / 2\rceil}\right) .\end{cases}
$$

For each integer $m$, let $\mathcal{D}_{p}(m)$ be the set of pairs $(a, b) \in \mathbb{F}_{p}^{*} \times \mathbb{F}_{p}^{*}$ such that $m \mid \# \mathbf{E}_{a, b}\left(\mathbb{F}_{p}\right)$. Then, by the result of Howe [29] we have the following asymptotic formula for $\# \mathcal{D}_{p}(m)$ :

Lemma 11. For all primes $p$ and all positive integers $m$, we have

$$
\left|\# \mathcal{D}_{p}(m)-\omega_{p}(m) p^{2}\right| \leqslant m^{1+o(1)} p^{3 / 2} \quad(m \rightarrow \infty) .
$$

\section{Main Results}

\subsection{Distribution of curves over finite fields}

For an arbitrary subset $\mathcal{S} \subseteq \mathbb{F}_{p} \times \mathbb{F}_{p}$, we denote by $M_{p}(\mathcal{S}, A, B)$ the number of curves $\mathbf{E}_{a, b}$ such that $(a, b) \in \mathcal{S},|a| \leqslant A$ and $|b| \leqslant B$. Here, we obtain an asymptotic formula for $M_{p}(\mathcal{S}, A, B)$. 
Similar to (7), we now define $e_{p}=\operatorname{gcd}(p-1,4)$ and put

$$
\rho_{p}(M)=\max _{\substack{\chi \in \mathcal{X}_{p}^{*} \\ \chi^{e}=\chi_{0}}}\left\{1,\left|\sum_{n=1}^{M} \chi(n)\right|\right\}
$$

We also denote

$$
\begin{gathered}
\mathcal{E}_{1}(A, B ; p)=\min \left\{A \sigma_{p}(B)+A^{1 / 2} B p^{1 / 4}+A^{1 / 2} B^{1 / 2} p^{1 / 2},\right. \\
\left.B \rho_{p}(A)+A B^{1 / 2} p^{1 / 4}+A^{1 / 2} B^{1 / 2} p^{1 / 2}\right\}, \\
\mathcal{E}_{2}(A, B ; p)=\min \left\{A \sigma_{p}(B)+B^{1 / 2} p \log p, B \rho_{p}(A)+A^{1 / 2} p \log p\right\} .
\end{gathered}
$$

Theorem 12. For all primes $p>3$, all integers $1 \leqslant A, B \leqslant(p-1) / 2$, and all subsets $\mathcal{S} \subseteq \mathbb{F}_{p} \times \mathbb{F}_{p}$ such that whenever $(r, s) \in \mathcal{S}$ and $\mathbf{E}_{a, b}\left(\mathbb{F}_{p}\right) \cong \mathbf{E}_{r, s}\left(\mathbb{F}_{p}\right)$ it follows that $(a, b) \in \mathcal{S}$, the following bound holds uniformly:

$$
M_{p}(\mathcal{S}, A, B)-\frac{4 A B}{p^{2}} \# \mathcal{S} \ll \min \left\{\mathcal{E}_{1}(A, B ; p) p^{o(1)}, \mathcal{E}_{2}(A, B ; p)\right\} .
$$

Proof. It follows from the properties of isomorphic curves given in Section 2.3 that

$$
M_{p}(\mathcal{S}, A, B)=\frac{1}{p-1} \sum_{(r, s) \in \mathcal{S}} \# \mathcal{Z}_{r, s}(A, B ; p)+O(A+B),
$$

where we have estimated the contribution from curves with $a b=0$ trivially as $O(A+B)$; note that if $a=r u^{4}$ and $b=s u^{6}$ then the same relations also hold with $-u$ instead of $u$, so each group $\mathbf{E}_{a, b}\left(\mathbb{F}_{p}\right)$ with $|a| \leqslant A$ and $|b| \leqslant B$ is counted precisely $p-1$ times in the sum on the right-hand side. Applying Corollary 6, we obtain that

$$
\begin{aligned}
M_{p}(\mathcal{S}, A, B)=\frac{4 A B}{p(p-1)} & \# \mathcal{S} \\
& \quad+O\left(A \sigma_{p}(B)+A^{1 / 2} B p^{1 / 4+o(1)}+A^{1 / 2} B^{1 / 2} p^{1 / 2+o(1)}\right) .
\end{aligned}
$$

Since

$$
\frac{4 A B}{p(p-1)} \# \mathcal{S}-\frac{4 A B}{p^{2}} \# \mathcal{S} \ll \frac{A B}{p} \leqslant A \leqslant A \sigma_{p}(B),
$$

it follows that

$$
M_{p}(\mathcal{S}, A, B)-\frac{4 A B}{p^{2}} \# \mathcal{S} \ll A \sigma_{p}(B)+A^{1 / 2} B p^{1 / 4+o(1)}+A^{1 / 2} B^{1 / 2} p^{1 / 2+o(1)} .
$$


Examining our arguments closely, in particular those of Section 2, we see that the roles of $A$ and $B$ are fully interchangeable, hence we also have

$$
M_{p}(\mathcal{S}, A, B)-\frac{4 A B}{p^{2}} \# \mathcal{S} \ll B \rho_{p}(A)+A B^{1 / 2} p^{1 / 4+o(1)}+A^{1 / 2} B^{1 / 2} p^{1 / 2+o(1)},
$$

and thus,

$$
M_{p}(\mathcal{S}, A, B)-\frac{4 A B}{p^{2}} \# \mathcal{S} \ll \mathcal{E}_{1}(A, B ; p) p^{o(1)} .
$$

Using Corollary 8 instead of Corollary 6, a similar argument shows that

$$
M_{p}(\mathcal{S}, A, B)-\frac{4 A B}{p^{2}} \# \mathcal{S} \ll \mathcal{E}_{2}(A, B ; p),
$$

and this concludes the proof.

Using Lemma 1 to estimate $\mathcal{E}_{1}(A, B ; p)$, we deduce that:

Corollary 13. Under the hypotheses of Theorem 12, the bound

$$
\begin{aligned}
& \left|M_{p}(\mathcal{S}, A, B)-\frac{4 A B}{p^{2}} \# \mathcal{S}\right| \\
& \leqslant \min \left\{A B^{1-1 / \nu} p^{(\nu+1) /\left(4 \nu^{2}\right)}+A^{1 / 2} B p^{1 / 4}+A^{1 / 2} B^{1 / 2} p^{1 / 2},\right. \\
& \left.A^{1-1 / \nu} B p^{(\nu+1) /\left(4 \nu^{2}\right)}+A B^{1 / 2} p^{1 / 4}+A^{1 / 2} B^{1 / 2} p^{1 / 2}\right\} p^{o(1)}
\end{aligned}
$$

holds with any fixed integer $\nu \geqslant 1$, where the function implied by o(1) depends only on $\nu$.

Similarly, using Lemma 1 to estimate $\mathcal{E}_{2}(A, B ; p)$ we obtain:

Corollary 14. Under the hypotheses of Theorem 12, the bound

$$
\begin{aligned}
& \left|M_{p}(\mathcal{S}, A, B)-\frac{4 A B}{p^{2}} \# \mathcal{S}\right| \\
& \quad \leqslant \min \left\{A B^{1-1 / \nu} p^{(\nu+1) /\left(4 \nu^{2}\right)}+B^{1 / 2} p, A^{1-1 / \nu} B p^{(\nu+1) /\left(4 \nu^{2}\right)}+A^{1 / 2} p\right\} p^{o(1)}
\end{aligned}
$$

holds with any fixed integer $\nu \geqslant 1$, where the function implied by o(1) depends only on $\nu$. 
Applying Corollary 13 with a sufficiently large integer $\nu$, we have the following:

Corollary 15. Under the hypotheses of Theorem 12, for any fixed $\varepsilon>0$ there exists $\delta>0$ such that if

$$
\min \{A, B\} \geqslant p^{1 / 4+\varepsilon} \quad \text { and } \quad A B \geqslant p^{1+\varepsilon},
$$

then

$$
M_{p}(\mathcal{S}, A, B)-\frac{4 A B}{p^{2}} \# \mathcal{S} \ll A B p^{-\delta},
$$

where the constant implied by $\ll$ depends only on $\varepsilon$.

\subsection{Sato-Tate conjecture on average}

Theorem 16. For any fixed $\varepsilon>0$ there exists $\delta>0$ such that for all integers $A$ and $B$ satisfying the inequalities (3) and (4), and all real numbers $0 \leqslant \alpha<\beta \leqslant \pi$, we have

$$
N_{\alpha, \beta}^{\mathrm{ST}}(A, B ; x)=\left(4 \mu_{\mathrm{ST}}(\alpha, \beta)+O\left(x^{-\delta}\right)\right) A B \pi(x),
$$

where the constant implied by $O$ depends only on $\varepsilon$.

Proof. Let us assume that $A \geqslant B$ since the case $A<B$ is similar. Using the trivial bound $M_{p}\left(\mathcal{T}_{p}(\alpha, \beta), A, B\right) \leqslant A B$ for primes $p \leqslant 2 A+1$, we have

$$
N_{\alpha, \beta}^{\mathrm{ST}}(A, B ; x)=\sum_{2 A+1<p \leqslant x} M_{p}\left(\mathcal{T}_{p}(\alpha, \beta), A, B\right)+O\left(A^{2} B\right) .
$$

Applying Lemma 9 and Theorem 12, we derive that

$$
M_{p}\left(\mathcal{T}_{p}(\alpha, \beta), A, B\right)-4 \mu_{\mathrm{ST}}(\alpha, \beta) A B \ll \mathcal{E}_{1}(A, B ; p) p^{o(1)}+A B p^{-1 / 4} .
$$

Therefore, since $\mu_{\mathrm{ST}}(\alpha, \beta) \ll 1$, we have

$$
N_{\alpha, \beta}^{\mathrm{ST}}(A, B ; x)-4 \mu_{\mathrm{ST}}(\alpha, \beta) A B \pi(x) \ll A^{2} B+A B x^{3 / 4}+x^{o(1)} \sum_{p \leqslant x} \mathcal{E}_{1}(A, B ; p) .
$$

Applying Lemma 3 with $\eta=1 / 24$, we get

$$
\sum_{p \leqslant x} \sigma_{p}(B) \leqslant B x^{11 / 12+o(1)}+B^{23 / 24} x \quad(x \rightarrow \infty),
$$


and it follows that

$$
\begin{aligned}
\sum_{p \leqslant x} \mathcal{E}_{1}(A, B ; p) \ll & A B x^{11 / 12+o(1)}+A B^{23 / 24} x \\
& +A^{1 / 2} B x^{5 / 4}+A^{1 / 2} B^{1 / 2} x^{3 / 2} \quad(x \rightarrow \infty) .
\end{aligned}
$$

After simple calculations, we obtain the stated result.

\subsection{Cyclicity on average}

Let $\Theta$ denote the following constant:

$$
\Theta=\prod_{q}\left(1-\frac{1}{q(q-1)\left(q^{2}-1\right)}\right)
$$

where the product runs over all primes $q$.

Theorem 17. Let $\varepsilon>0$ and $K>0$ be fixed. Then, for all integers $A$ and $B$ satisfying the inequalities (3) and (4), we have

$$
N^{\mathrm{C}}(A, B ; x)=\left(4 \Theta+O\left((\log x)^{-K}\right)\right) A B \pi(x),
$$

where the constant implied by $O$ depends only on $\varepsilon$ and $K$.

Proof. Let us assume that $A \geqslant B$ since the case $A<B$ is similar. Using the trivial bound $M_{p}\left(\mathcal{C}_{p}, A, B\right) \leqslant A B$ for primes $p \leqslant 2 A+1$, we have

$$
N^{\mathrm{C}}(A, B ; x)=\sum_{2 A+1<p \leqslant x} M_{p}\left(\mathcal{C}_{p}, A, B\right)+O\left(A^{2} B\right) .
$$

Applying Lemma 10 and Theorem 12, we derive that

$$
\left|M_{p}\left(\mathcal{C}_{p}, A, B\right)-4 \vartheta_{p} A B\right| \leqslant\left(\mathcal{E}_{1}(A, B ; p)+A B p^{-1 / 2}\right) p^{o(1)} .
$$

Since the bound $A B \leqslant p^{2}$ implies $A B p^{-1 / 2} \leqslant A^{1 / 2} B^{1 / 2} p^{1 / 2}$, and the second term can be dropped; thus,

$$
\left|M_{p}\left(\mathcal{C}_{p}, A, B\right)-4 \vartheta_{p} A B\right| \leqslant \mathcal{E}_{1}(A, B ; p) p^{o(1)} .
$$

Hence, using (12), the inequality $A^{2} B \leqslant A B x /(\log x)^{K+1}$, and the trivial bound $\vartheta_{p} \ll 1$ for primes $p \leqslant 2 A+1$, after simple calculations we derive the estimate

$$
N^{\mathrm{C}}(A, B ; x)=4 A B \sum_{p \leqslant x} \vartheta_{p}+O\left(\frac{A B x}{(\log x)^{K+1}}\right) .
$$


Now write

$$
\sum_{p \leqslant x} \vartheta_{p}=\sum_{p \leqslant x} f(p-1)
$$

where

$$
f(n)=\prod_{q \mid n}\left(1-\frac{1}{q\left(q^{2}-1\right)}\right)
$$

the product being taken over all prime divisors $q$ of $n$; note that $f(n)$ is a multiplicative function. Let $g(n)$ be the multiplicative function that is defined on prime powers $q^{k}$ as follows:

$$
g\left(q^{k}\right)= \begin{cases}\frac{-1}{q\left(q^{2}-1\right)} & \text { if } k=1 \\ 0 & \text { if } k \geqslant 2\end{cases}
$$

Then,

$$
f(n)=\sum_{d \mid n} g(d)
$$

It is easy to check that the functions $f(n)$ and $g(n)$ satisfy the conditions of [30, Theorem 3], hence it follows that

$$
\sum_{p \leqslant x} \vartheta_{p}=\sum_{p \leqslant x} f(p-1)=\Theta \pi(x)+O\left(\frac{x}{(\log x)^{K+1}}\right)
$$

with

$$
\Theta=\sum_{n=1}^{\infty} \frac{g(d)}{\varphi(d)}=\prod_{q}\left(1-\frac{1}{q(q-1)\left(q^{2}-1\right)}\right) .
$$

Inserting the estimate (14) into (13), we finish the proof.

\subsection{Divisibility on average}

Put

$$
\mu=\prod_{q^{j} \| m} q^{\lceil j / 2\rceil}
$$

and set

$$
\Omega_{m}=\frac{1}{\varphi(\mu)} \sum_{\substack{1 \leqslant k \leqslant \mu \\ \operatorname{gcd}(k, \mu)=1}} \omega_{k}(m)
$$


where $\varphi(\mu)$ is the Euler function, and $\omega_{k}(m)$ is the completely multiplicative function which is defined on prime powers $q^{j}$ by (11). For example, if $m=q$ is prime, then we have

$$
\Omega_{q}=\frac{1}{q-1}\left(\frac{q}{q^{2}-1}+\sum_{k=2}^{q-1} \frac{1}{q-1}\right)=\frac{q^{2}-2}{(q-1)\left(q^{2}-1\right)} .
$$

Theorem 18. Let $\varepsilon>0$ and $K>0$ be fixed. Then, for all integers $A$ and $B$ satisfying the inequalities (3) and (4), and all integers $m \leqslant(\log x)^{K}$, we have

$$
N_{m}^{\mathrm{D}}(A, B ; x)=\left(4 \Omega_{m}+O\left((\log x)^{-K}\right)\right) A B \pi(x),
$$

where the constant implied by $O$ depends only on $K$ and $\varepsilon$.

Proof. Let us assume that $A \geqslant B$ since the case $A<B$ is similar. Using the trivial bound $M_{p}\left(\mathcal{D}_{p}(m), A, B\right) \leqslant A B$ for primes $p \leqslant 2 A+1$, we have

$$
N_{m}^{\mathrm{D}}(A, B ; x)=\sum_{2 A+1<p \leqslant x} M_{p}\left(\mathcal{D}_{p}(m), A, B\right)+O\left(A^{2} B\right) .
$$

Applying Lemma 11 and Theorem 12, we see that for $p \leqslant x$ and $x \rightarrow \infty$ :

$$
\begin{aligned}
\left|M_{p}\left(\mathcal{D}_{p}(m), A, B\right)-4 \omega_{p}(m) A B\right| & \leqslant\left(\mathcal{E}_{1}(A, B ; p)+A B p^{-1 / 2}\right) p^{o(1)} \\
& \leqslant \mathcal{E}_{1}(A, B ; p) p^{o(1)}
\end{aligned}
$$

where the second inequality follows from the fact that the term $A B p^{-1 / 2}$ never dominates $\mathcal{E}_{1}(A, B ; p)$ (see the proof of Theorem 17). Hence, using (12) and the trivial bound $\omega_{p}(m) \ll 1$ for primes $p \leqslant 2 A+1$, we conclude that

$$
\begin{aligned}
N_{m}^{\mathrm{D}}(A, B ; x)=4 A B & \sum_{p \leqslant x} \omega_{p}(m) \\
+O & \left(A B x^{11 / 12+o(1)}+A B^{23 / 24} x\right. \\
& \left.+A^{1 / 2} B x^{5 / 4+o(1)}+A^{1 / 2} B^{1 / 2} x^{3 / 2+o(1)}+A^{2} B\right) .
\end{aligned}
$$

As the value of $\omega_{p}(m)$ depends only on the residue class of $p$ modulo $\mu$, where $\mu$ is given by (15), using the Siegel-Walfisz theorem (see [31, Corollary 5.29]) we immediately obtain the desired result. 


\section{Primes $p$ with $\operatorname{gcd}(p-1,12)=2$}

Clearly, Theorem 12 leads to nontrivial results only under the condition $\max \{A, B\} \geqslant p^{1 / 4+\varepsilon}$ which is determined by Lemma 1 (this is where the relevant character sums admit nontrivial estimates). However in the special case that $\operatorname{gcd}(p-1,12)=2$, using the result of [7] on the density of quadratic residues and nonresidues in short intervals, one can obtain some nontrivial estimates over a wider range.

Indeed, if $\operatorname{gcd}(p-1,6)=2$, then

$$
\begin{aligned}
\mathcal{Z}_{s}(B ; p) & =\left\{u \in \mathbb{F}_{p}^{*}: s u^{6} \equiv b \quad(\bmod p) \text { where }|b| \leqslant B\right\} \\
& =\left\{u \in \mathbb{F}_{p}^{*}: s u^{2} \equiv b \quad(\bmod p) \text { where }|b| \leqslant B\right\} .
\end{aligned}
$$

Thus $\mathcal{Z}_{s}(B ; p) / 2$ is the number of quadratic residues or nonresidues in the interval $|b| \leqslant B$ (according to whether $s$ is a quadratic residue or nonresidue). Therefore, by the result of [7] we have

$$
\mathcal{Z}_{s}(B ; p) \gg B
$$

whenever $B \geqslant p^{1 /(4 \sqrt{e})+\varepsilon}$ for some fixed $\varepsilon$ and all sufficiently large $p$. This new bound can be used in Lemmas 5 and 7 as before. If $\operatorname{gcd}(p-1,4)=2$ as well, then similar arguments can be applied with respect to $A$. Arguing as in the proof of Theorem [12, we obtain a lower bound on $M_{p}(\mathcal{S}, A, B)$ which holds under the condition

$$
\min \{A, B\} \geqslant p^{1 /(4 \sqrt{e})+\varepsilon} \quad \text { and } \quad A B \geqslant p^{1+\varepsilon} .
$$

\section{$5 \quad$ Further Applications}

For specific ranges of the parameters $A$ and $B$, one can use Lemma 1 instead of (or in conjunction with) Lemma 3 to obtain stronger and more explicit bounds for the error term in Theorem [16. On the other hand, in Theorems 17] and 18 the main contribution to the error comes from the imprecision involved in estimating sums with $\vartheta_{p}$ and $\omega_{p}(m)$, respectively.

Using Lemma 1 in place of Lemma 3 also allows one to study averages in which the parameters $a$ and $b$ vary over the shifted intervals $[H+1, H+K]$ and $[L+1, L+M]$, respectively. 
Our arguments can also be used to improve the bound of [8] on the size of the "smallest" Weierstraß equation which is isomorphic to a given curve E. Given an elliptic curve $\mathbf{E}$ over $\mathbb{F}_{p}$ let us define

$$
\mu(\mathbf{E})=\min \left\{\max \{a, b\}: 1 \leqslant a, b<p, \mathbf{E}_{a, b} \cong \mathbf{E}\right\},
$$

(that is, the minimum is taken over all curves $\mathbf{E}_{a, b}$ which are isomorphic to $\mathbf{E})$. This question has been recently considered in [12], where, using a variant of the method of [23] the bound $\mu(E)=O\left(p^{3 / 4}\right)$ has been derived. It has been shown in [8] that for almost all curves one has $\mu(\mathbf{E}) \leqslant p^{2 / 3+o(1)}$. Using a variant of Theorem 12 for the intervals $1 \leqslant a \leqslant A$ and $1 \leqslant b \leqslant B$ one easily derives $\mu(\mathbf{E}) \leqslant p^{1 / 2+o(1)}$ for almost all curves $\mathbf{E}$ over $\mathbb{F}_{p}$.

Here, we have not used the full strength of the results of Garaev 25]. Doing so, one can actually replace the lower bound $A, B \geqslant x^{\varepsilon}$ in (3) with the bound $A, B \geqslant \exp (c \sqrt{\log x})$ for an appropriate constant $c>0$ and drop the condition (4).

For fixed integers $m>k \geqslant 0$, one can also study the counting functions $\varpi_{a, b}^{(E)}(m, k ; x)$ and $\varpi_{a, b}^{(t)}(m, k ; x)$ of primes $p \leqslant x$ (with $\left.p \nmid 4 a^{3}+27 b^{2}\right)$ such that

$$
\# \mathbf{E}_{a, b}\left(\mathbb{F}_{p}\right) \equiv k \quad(\bmod m) \quad \text { and } \quad p+1-\# \mathbf{E}_{a, b}\left(\mathbb{F}_{p}\right) \equiv k \quad(\bmod m),
$$

respectively. Our method can be adapted to obtain asymptotic formulae for the average values

$$
\frac{m}{8 A B} \sum_{|a| \leqslant A} \sum_{|b| \leqslant B} \varpi_{a, b}^{(E)}(m, k ; x) \quad \text { and } \quad \frac{m}{8 A B} \sum_{|a| \leqslant A} \sum_{|b| \leqslant B} \varpi_{a, b}^{(t)}(m, k ; x)
$$

over a wide range of values in the parameters $A, B$ and $m$.

In principle, one can combine our approach with the results of [38] to study the distribution of the discriminants of complex multiplication fields of the curves $\mathbf{E}_{a, b}\left(\mathbb{F}_{p}\right)$, on average over $a$ and $b$. Such discriminants are related to the size of the Tate-Shafarevich group of $\mathbf{E}_{a, b}\left(\mathbb{F}_{p}\right)$; thus, it is likely that our approach can be used to improve some of the estimates of [16] on average.

We remark that the distribution of prime values of $\# \mathbf{E}_{a, b}\left(\mathbb{F}_{p}\right)$ is of great interest in the theory of cryptography. Our method can be adapted to study this question as $a$ and $b$ vary over short intervals; see [6], where also the challenging task of evaluating the main term has been treated.

For the problems mentioned above, the corresponding sets of curves are "massive" enough to permit an application of Theorem 12, nevertheless, the 
main obstacle in each case is the evaluation of the main term, which may require a significant effort even if the work is feasible.

It is natural to try to combine our approach with recent work of James and $\mathrm{Yu}[33$ ] which studies, on average over $|a| \leqslant A$ and $|b| \leqslant B$, the number of primes $p \leqslant x$ for which $p+1-\mathbf{E}_{a, b}\left(\mathbb{F}_{p}\right)$ is a perfect $k$-th power. In some cases, it may be possible to lower the threshold on $A$ and $B$. For $k \geqslant 3$ the corresponding set of curves appears to be too sparse, but perhaps for $k=2$ there is a chance for our method to yield an improvement.

\section{References}

[1] A. Akbary, C. David and R. Juricevic, 'Average distributions and products of special values of L-series', Acta Arith. 111 (2004), 239268.

[2] A. Ayyad, T. Cochrane and Z. Zheng, 'The congruence $x_{1} x_{2} \equiv x_{3} x_{4}$ $(\bmod p)$, the equation $x_{1} x_{2}=x_{3} x_{4}$ and the mean value of character sums', J. Number Theory 59 (1996), 398-413.

[3] S. Baier, 'The Lang-Trotter conjecture on average', J. Ramanujan Math. Soc., (to appear).

[4] S. Baier, 'A remark on the conjectures of Lang-Trotter and Sato-Tate on average', Preprint, 2007.

[5] S. Baier and L. Zhao, 'The Sato-Tate conjecture on average for small angles', Trans. Am. Math. Soc., (to appear).

[6] A. Balog, A. Cojocaru and C. David, 'Average twin prime conjecture for elliptic curves', Preprint, 2007.

[7] W. D. Banks, M. Z. Garaev, D. R. Heath-Brown and I. E. Shparlinski, 'Density of non-residues in Burgess-type intervals and applications', Bull. Lond. Math. Soc., (to appear).

[8] W. D. Banks and I. E. Shparlinski, 'Average normalizations of elliptic curves', Bull. Austral. Math. Soc. 66 (2002), 353-358. 
[9] J. Battista, J. Bayless, D. Ivanov and K. James, 'Average Frobenius distributions for elliptic curves with nontrivial rational torsion', Acta Arith. 119 (2005), 81-91.

[10] B. J. Birch, 'How the number of points of an elliptic curve over a fixed prime field varies', J. Lond. Math. Soc. 43 (1968), 57-60.

[11] I. Borosh, C. J. Moreno and H. Porta, 'Elliptic curves over finite fields. II', Math. Comput. 29 (1975), 951-964.

[12] M. Ciet, J.-J. Quisquater and F. Sica, 'Elliptic curve normalization', Crypto Group Technical Report Series CG-2001/2, Univ. Catholique de Louvain, Belgium, 2001, 1-13.

[13] A. Cojocaru, 'On the cyclicity of the group of $\mathbb{F}_{p}$-rational points of non-CM elliptic curves', J. Number Theory 96 (2002), 335-350.

[14] A. Cojocaru, 'Cyclicity of CM elliptic curves modulo p', Trans. Amer. Math. Soc. 355 (2003), 2651-2662 (electronic).

[15] A. Cojocaru, 'Questions about the reductions modulo primes of an elliptic curve', in Number theory, 61-79, CRM Proc. Lecture Notes, 36, Amer. Math. Soc., Providence, RI, 2004.

[16] A. Cojocaru and W. Duke, 'Reductions of an elliptic curve and their Tate-Shafarevich groups', Math. Ann. 329 (2004), 513-534.

[17] A. Cojocaru and M. R. Murty, 'Cyclicity of elliptic curves modulo $p$ and elliptic curve analogues of Linnik's problem', Math. Ann. 330 (2004), 601-625.

[18] A. Cojocaru, É. Fouvry and M. R. Murty, 'The square sieve and the Lang-Trotter conjecture', Canad. J. Math. 57 (2005), 1155-1177.

[19] C. David and F. Pappalardi, 'Average Frobenius distributions of elliptic curves', Internat. Math. Res. Notices 1999, 165-183.

[20] C. David and F. Pappalardi, 'Average Frobenius distribution for inerts in $\mathbb{Q}(i)^{\prime}$, J. Ramanujan Math. Soc. 19 (2004), 181-201.

[21] W. Duke, 'Elliptic curves with no exceptional primes', C. R. Acad. Sci., Paris, Sér. I, Math. 325 (1997), 813-818. 
[22] B. Fisher, 'Equdistribution theorems (d'après P. Deligne amd N. Katz)', in Columbia University Number Theory Seminar (New York, 1992), Astérisque, vol. 228, Soc. Math. France, 1995, 69-79.

[23] É. Fouvry and M. R. Murty, 'On the distribution of supersingular primes', Canad. J. Math. 48 (1996), 81-104.

[24] J. B. Friedlander and H. Iwaniec, 'The divisor problem for arithmetic progressions', Acta Arith. 45 (1985), 273-277.

[25] M. Z. Garaev, 'Character sums in short intervals and the multiplication table modulo a large prime', Monat. Math. 148 (2006), 127-138.

[26] E.-U. Gekeler, 'Frobenius distributions of elliptic curves over finite prime fields', Int. Math. Res. Not. 2003, 1999-2018.

[27] D. Grant, 'A formula for the number of elliptic curves with exceptional primes, Compos. Math. 122 (2000), 151-164.

[28] R. Gupta and M. R. Murty, 'Cyclicity and generation of points mod $p$ on elliptic curves', Invent. Math. 101 (1990), 225-235.

[29] E. W. Howe, 'On the group orders of elliptic curves over finite fields, Compositio Math. 85 (1993), 229-247.

[30] K.-H. Indlekofer, S. Wehmeier and L. G. Lucht, 'Mean behaviour and distribution properties of multiplicative functions', Comput. Math. Appl. 48 (2004), 1947-1971.

[31] H. Iwaniec and E. Kowalski, Analytic number theory, American Mathematical Society, Providence, RI, 2004.

[32] K. James, 'Average Frobenius distributions for elliptic curves with 3-torsion', J. Number Theory 109 (2004), 278-298.

[33] K. James and G. Yu, 'Average Frobenius distribution of elliptic curves', Acta Arith. 124 (2006), 79-100.

[34] N. M. Katz, Gauss sums, Kloosterman sums, and monodromy groups, Princeton Univ. Press, Princeton, NJ, 1988. 
[35] S. Lang and H. Trotter, 'Frobenius distributions in $\mathrm{GL}_{2}$-extensions', in Lecture Notes in Mathematics, Vol. 504. Springer-Verlag, Berlin-New York, 1976.

[36] H. W. Lenstra, 'Factoring integers with elliptic curves', Ann. of Math. (2) 126 (1987), 649-673.

[37] R. Lidl and H. Niederreiter, Finite fields, Cambridge University Press, Cambridge, 1997.

[38] F. Luca and I. E. Shparlinski, 'Discriminants of complex multiplication fields of elliptic curves over finite fields', Canad. Math. Bull. 50 (2007), 409-417.

[39] M. R. Murty, 'On Artin's conjecture', J. Number Theory 16 (1983), 147-168.

[40] M. R. Murty and I. E. Shparlinski, 'Group structure of elliptic curves over finite fields and applications', in Topics in Geometry, Coding Theory and Cryptography, Springer-Verlag, 2006 (to appear).

[41] V. K. Murty, 'On the Sato-Tate conjecture', in Number Theory Related to Fermat's Last Theorem (Cambridge, Mass., 1981), Birkhäuser, Boston, Mass., 1982, 195-205.

[42] H. Niederreiter, 'The distribution of values of Kloosterman sums', Arch. Math. 56 (1991), 270-277.

[43] J.-P. Serre, 'Résumé des cours de 1977-1978', in Collected Papers, Vol. III, 465-468, Springer Verlag, Berlin, 1986.

[44] J. H. Silverman, The arithmetic of elliptic curves, Springer-Verlag, Berlin, 1995.

[45] R. Taylor, 'Automorphy for some $l$-adic lifts of automorphic mod $l$ representations, II', Preprint, 2006.

[46] S. G. Vlădut, 'Cyclicity statistics for elliptic curves over finite fields', Finite Fields Appl. 5 (1999), 13-25. 